\title{
PRINCIPLES AND TOOLS FOR TESTING OPEN DISTRIBUTED SYSTEMS *
}

\author{
Mohammed Benattou, Leo Cacciari, Régis Pasini and Omar Rafiq \\ Laboratoire TASC, University of Pau, France
}

\begin{abstract}
With emergence of new models, architectures and midlleware such as ODP, TINA and CORBA for developing open distributed systems, testing technology requires adaptation for use within conformance assessment in such systems. All these frameworks are object-based and aim at creating open distributed environments supporting interworking, interoperability, and portability, in spite of heterogeneity and autonomy of the related systems. In this context an open distributed system may be viewed as a system providing standardized distributed interfaces for interacting with other systems. Conformance of such a system can be assessed by attaching a related tester at each provided interface. However, many problems of controllability and observability influencing fault detection during the testing process arise if there is no coordination between the testers. In this paper, we show how to cope with these problems by using a distributed test method derived from OSI conformance testing. Afterwards, a CORBA prototype is designed, realized and experimented with a forum application.
\end{abstract}

\section{INTRODUCTION}

ODP (Open Distributed Processing) [3], is a joint ISO/ITU standard which provides a generic framework for building open distributed systems. CORBA (Common Object Request Broker Architecture) [7], which is a more implementation-oriented architecture with similar objectives to those of ODP, has been defined by the international consortium OMG (Object Management Group). Several implementations of CORBA are already commercially available. TINA (Telecommunications Information Networking Architecture) [10], designed by the international Consortium TINA-C, is a kind of future telecommunications architecture based on ODP and CORBA. ODP, CORBA and TINA are then

*This work has been supported by CNET-France Télécom under Grant 98 1B 221 as part of the CTI programme. 
complementary frameworks and they are currently recognized as constituting a real trilogy of open distributed processing.

In this context an open distributed system may be viewed as a system providing standardized distributed interfaces for interacting with other systems. To deal with this interfacing problem in conformance testing, ODP has already defined the concept of reference point as part of the reference model. It is a specific location of an open distributed system at which one or more interfaces can be localized, so that interactions at these interfaces can be observed. Hence, some subset or all possible reference points may be declared as conformance points, stating the requirements to be met at each of them. Some other work has also been done on the subject $[1,5,6]$; however, much effort and considerable work are required before reaching a workable and accepted conformance testing methodology for open distributed processing.

Based on testing of OSI communicating systems, conformance of an open distributed system can be assessed by attaching a related tester at each provided interface. However many problems of controllability and observability, influencing fault detection during the testing process arise if there is no coordination between the testers. In this paper, we show how to cope with these problems by using a distributed test method derived from OSI conformance testing [2, 9]. Afterwards, a CORBA prototype is designed, realized and experimented with a forum application.

\section{TESTING DISTRIBUTED APPLICATIONS}

Concurrent with academic research activities, efforts have been made within industry and standardization bodies (ISO and ITU) since the 80s to develop approaches for conformance testing of OSI protocols. After a decade of hard work, a standardized methodology, tools, experiences, and theories exist today with a certain degree of maturity [4]. This section is dedicated to extending results from protocol testing to deal with testing of an implementation under test (IUT) of an open distributed system.

\subsection{TESTING ARCHITECTURE}

Testing may be seen as a means to execute an IUT by carrying out test cases, in order to observe whether it has a certain behavior or not. An IUT is a kind of black-box with points of control and observation (PCOs) at which control of input events and observation of output events may take place during the testing process.

Testing architecture is a description of the environment within which an IUT is tested. Figure 1 gives an abstract view of what could be an ODP testing architecture. It is a synthesis of the architectures that have been used or proposed until now in the context of testing ODP systems $[1,5,6]$. It consists of 
the IUT, its PCOs that could be reference points or not, and a test system which may be seen as an implementation of test cases. The test system has particularly to execute given test cases by communicating with the IUT via its PCOs and to observe the test results. It may consist of one or several components called testers. The example of Figure 1 is made up of three testers.

Dealing with this architecture similarly to OSI conformance testing methodology [2], one can distinguish four kinds of test method that could be defined in terms of coordination between the testers in the testing process. If the test system consists of one tester, the related test method is called a centralized test method. If it consists of several testers located at different sites, we have three cases. If there is no coordination between the testers and each of them behaves with its corresponding PCO, independently of the others, the method is called a remote test method. If the testing process has to deal with the coordination of the testers, the related method is called a distributed test method. Finally, if the coordination has to follow given specific procedures, the method is called a coordinated test method and therefore is a particular case of the distributed one.

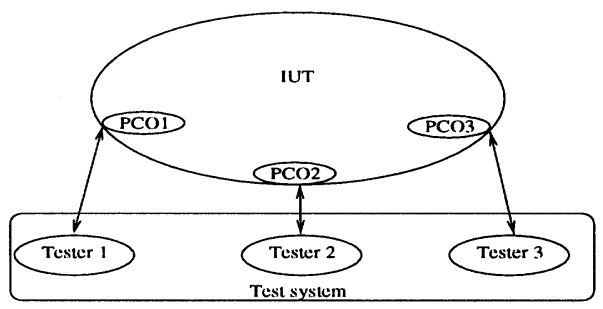

Figure 1. ODP testing architecture

\subsection{MODELING CONCEPTS}

Generally speaking, specifications and related implementations are described in different formalisms. In order to be able to reason about the testing process in a formal setting, the specification and IUT must be modeled by comparable concepts. Therefore, conformance of an IUT to its specification may be defined by means of relations between the IUT model and specification model [4].

I/O FSM (Input/Output Finite State Machines) are widely used in the communication protocol area [4], and may be easily adapted with some extensions for modeling distributed systems. In a communication protocol, a protocol entity communicating with a peer entity, is described by an I/O FSM with one input queue and one output queue. Distributed applications are supposed however to communicate with multiple partners. This leads to the notion of a multi-port FSM [6] which may use several input/output queues called ports. 
Definition 1. A multi-port FSM with $n$ ports (np-FSM) $\mathcal{A}$ is a 6-tuple $\left(Q, \Sigma, \Gamma, \delta, \lambda, q_{0}\right)$, where: $Q$ is the finite set of states of $\mathcal{A} ; q_{0} \in Q$ is a distinguished state, the initial state of $\mathcal{A} ; \Sigma$ is a n-tuple $\left(\Sigma_{1}, \Sigma_{2}, \ldots, \Sigma_{n}\right)$ where $\Sigma_{k}$ is the input alphabet of port $k$, and $\Sigma_{i} \cap \Sigma_{j}=\emptyset$ for $i \neq j$. We write $\bar{\Sigma}$ for the input alphabet $\Sigma_{1} \cup \Sigma_{2} \cup \cdots \cup \Sigma_{n}$ of $\mathcal{A} ; \Gamma$ is a n-tuple $\left(\Gamma_{1}, \Gamma_{2}, \ldots, \Gamma_{n}\right)$ where $\Gamma_{k}$ is the output alphabet of port $k$, and $\Gamma_{i} \cap \Gamma_{j}=\emptyset$ if $i \neq j$. $\varepsilon$ being the empty symbol, we write $\vec{\Gamma}$ for the output alphabet $\left(\Gamma_{1} \cup\{\varepsilon\}\right) \times\left(\Gamma_{2} \cup\{\varepsilon\}\right) \times \cdots \times\left(\Gamma_{n} \cup\{\varepsilon\}\right)$ of $\mathcal{A} ; \delta$ is the transition function, it is a partial function $Q \times \bar{\Sigma} \rightarrow Q ; \lambda$ is the output function, it is a partial function $Q \times \bar{\Sigma} \rightarrow \vec{\Gamma}$. Moreover, $\lambda(q, \alpha)$ is defined if and only if $\delta(q, \alpha)$ is.

$A$ transition of np-FSM $\mathcal{A}$ is a 4-tuple $\mathbf{t}=\left(q, \alpha, \gamma, q^{\prime}\right)$ where $q, q^{\prime} \in Q$, $\alpha \in \bar{\Sigma}$ and $\gamma \in \vec{\Gamma}$ are such that $\delta(q, \alpha)=q^{\prime}$ and $\lambda(q, \alpha)=\gamma$.

Example 1. Figure 2 gives an example of 3p-FSM with set state $Q=\left\{q_{0}, q_{1}\right.$, $\left.q_{2}, q_{3}, q_{4}, q_{5}\right\}, q_{0}$ being the initial state, $\Sigma_{1}=\{a\}, \Sigma_{2}=\{b\}, \Sigma_{3}=\{c\}$, and $\Gamma_{1}=\{w, x\}, \Gamma_{2}=\{y\}, \Gamma_{3}=\{z\}$. Transitions are represented by arrows.

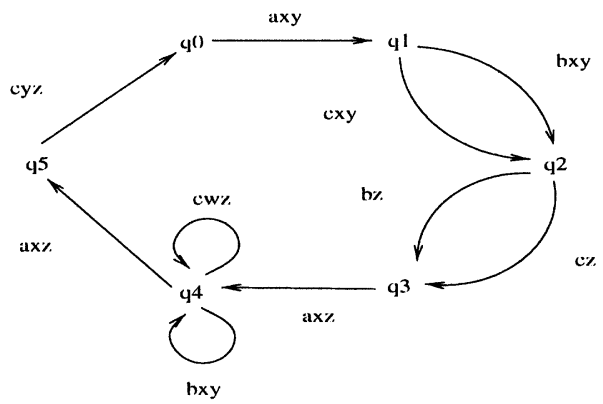

Figure 2. An example of 3p-FSM

Definition 2. $A$ test sequence of np-FSM $\mathcal{A}$ is a sequence $\omega$ in the form: $! x_{1} ? y_{1} ! x_{2} ? y_{2} \cdots ! x_{t} ? y_{t}$ where, for $i=1,2, \ldots, t, x_{i}$ belongs to $\bar{\Sigma}$ and $y_{i}$ is a subset of $\cup_{k=1}^{n} \Gamma_{k}$ such that, for each port $k,\left|y_{i} \cap \Gamma_{k}\right| \leq 1$, i.e. $y_{i}$ contains at most one symbol from the output alphabet of each port of $\mathcal{A}$. ! $x_{i}$ means sending message $x_{i}$ to the IUT and ? $y_{i}$ means receiving the messages belonging to $y_{i}$ from the IUT.

Example 2. A test sequence for Example 1:

$$
\omega=! a ?\{x, y\} ! b ?\{x, y\} ! c\{z\} ! a ?\{x, z\} ! b ?\{x, y\} ! c ?\{w, z\} ! a ?\{x, z\} ! c ?\{y, z\}
$$

Test sequences are generally generated from the IUT specification and characterized by their fault coverage. Several methods exist for generating test 
sequences from I/O FSM specifications. They are mainly for detecting two basic types of fault: output faults and transfer faults [8]. An edge with an incorrect output has an output fault which is generally observable. Any generation method providing a test suite traversing each transition of an FSM at least once is capable of detecting such faults. An edge with an incorrect starting state or ending state has a transfer fault. Since states are not directly observable, these faults are relatively more difficult to detect. No specific test generation method is considered in the following. Test sequences may be any.

\subsection{CONTROLLABILITY AND OBSERVABILITY ISSUES}

If the centralized method does not pose any particular problem, the others raise several issues in terms of controllability and observability which are fundamental features of conformance testing. In this context, controllability may be defined as the testing power or the capability of the test system to realize input events at corresponding PCOs in a given order, and observability may be defined as the testing power or the capability of the test system to determine the output events and the order in which they take place at the corresponding PCOs.

It is easy to see that controllability and observability related to the chosen test method have a great influence on several aspects of the testing activity such as the execution of test sequences, the observations that can be made during the test execution, and the interpretation of the testing results.

Example 3. Figure 3 gives an example of faulty IUT related to Example 1. It is easy to see that test sequence (1) in the centralized method leads to a fail verdict. In the remote method however projections of (1) on port alphabets are required to get the test sequence related to each tester. Projections $\omega_{1}$, $\omega_{2}$ and $\omega_{3}$ of $\omega$ on port 1 , port 2 and port 3 are: $\omega_{1}=! a ? x ? x ! a ? x ? x ? w ! a ? x$, $\omega_{2}=? y ! b ? y ! b ? y ? y$ and $\omega_{3}=! c ? z ? z ! c ? z ? z ! c ? z$.

Let us examine three situations in applying these sequences to the IUT of Figure 3 by using the remote method. The test starts in $q_{0}$.

Situation 1. When the IUT is in $q_{4}$, it gets both $b$ on port 2 and $c$ on port 3 . Then, either it follows the intended path reading $b$ before $c$, or it reads $c$ before $b$. In the first case, tester 1 receives $w$ before $x$ and it detects an output fault. However, if the IUT decides to read $c$ before $b$ then none of the testers is able to detect this fault.

Situation 2. When the IUT is in $q_{4}$, it receives $a$ and then it sends $y$ and $z$ instead of $x$ and $z$ and goes to $q_{5}$. In $q_{5}$, it receives $c$ and sends $x$ and $z$ instead of $y$ and $z$ and goes to $q_{0}$. One can notice that there are two successive output faults and none of the testers is able to detect them. 


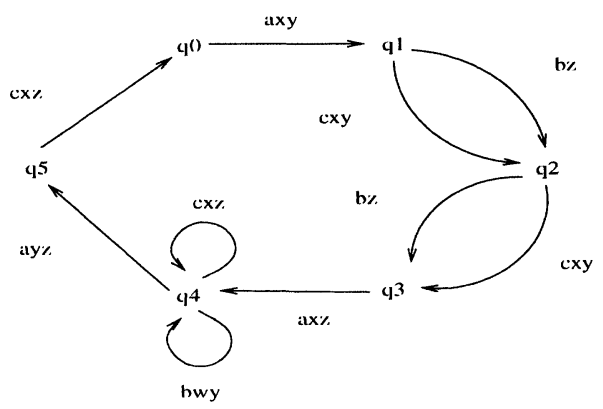

Figure 3. Example of faulty IUT for Example 1

Situation 3. When the IUT is in $q_{1}$, it gets both $b$ on port 2 and $c$ on port 3 . Then, either it follows the intended path reading $b$ before $c$, or it reads $c$ before $b$. In both cases, tester 1 receives $x$, tester 2 receives $y$ and tester 3 receives $z$ as specified by the test sequences of Example 3. However, one can notice that the IUT has in fact two successive output faults: on receiving $b$ in $q_{1}$, IUT sends $z$ instead of $x$ and $y$ and on receiving $c$ in $q_{2}$, IUT sends $x$ and $y$ instead of $z$.

In situation 1, there is a control problem because the input events $b$ and $c$ may be taken into account by the IUT in any order. In situation 2 , there is an observation problem as the testers are unable to determine the order in which output events take place. Finally, in situation 3, there is both a control problem and an observation problem. All these situations have already been analyzed in [6]. They are due to the fact that, in the remote method, there is no relationship between the testers except the IUT itself. This implies, among others, that the fault coverage of a test sequence may be lower in the remote method than in the centralized one.

\section{DISTRIBUTED TEST METHOD}

In this section, we are designing a configuration for the distributed method that is able to avoid control and observation problems by using auxiliary messages called coordination messages.

\subsection{ARCHITECTURE}

The basic idea is to coordinate the testers by using a communication service parallel to the IUT through a multicast channel. Each tester interacts with the IUT only through the port to which it is attached and communicates with the other testers through the multicast channel. It is an extension of the Astride approach that we have already designed for protocol testing [9].

The multicast channel is mainly to allow each tester to send a coordination message to the other testers during testing. We are aware of the importance 
of temporal considerations and their coherence in testing distributed systems. To cope with that in this contribution, we make a simplifying and realistic hypothesis. We assume that the time required for a coordination message to travel from a tester to another tester is of the same order of magnitude as the reaction time of the IUT, i.e. the time elapsed between the reception of a message by the IUT and the sending by the IUT of the corresponding response. Figure 4 depicts an architecture of our distributed test method.

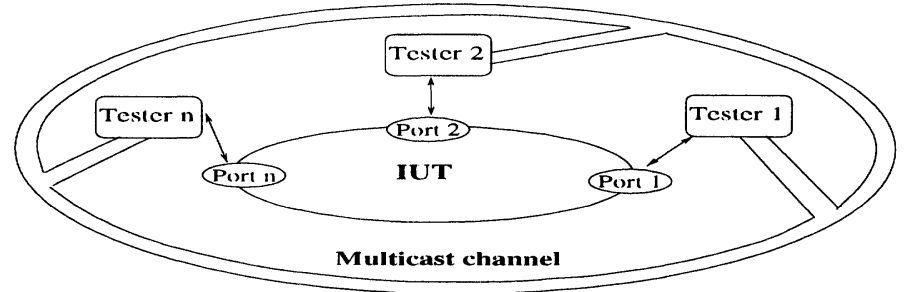

Figure 4. Distributed test architecture

\subsection{LOCAL TEST SEQUENCES}

In the distributed test method, each tester executes a local test sequence constructed from the complete test sequence of the IUT. A local test sequence is in the form $\alpha_{1} \alpha_{2} \cdots \alpha_{t}$, where each $\alpha_{i}$ is either:

- ! $x$, sending of message $x \in \Sigma_{k}$ to the IUT,

- ? $y$, receiving of message $y \in \Gamma_{k}$ from the IUT,

- $-c_{h_{1}, \ldots, h_{r}}$, sending of coordination message $c$ to testers $h_{1}, \ldots, h_{r}$,

- $+c_{h}$, receiving of coordination message $c$ from tester $h$.

For each $\alpha_{i}$, if it is a sending, either of a message to the IUT or of a coordination message, the tester sends it. If $\alpha_{i}$ is a receiving, either of an output from IUT or of a coordination message, then the tester waits for a message. If no message is received, or if the received message is not the expected one, the tester gives a fail verdict. If the tester reaches the end of its sequence, then it gives a pass verdict. If all testers give a pass verdict, then the test system ends the test by giving a pass verdict.

If the IUT has $n$ ports, Algorithm 1 is dedicated to compute the $n$ related local test sequences from a complete test sequence of IUT. $\backslash$ is to denote the set difference and $\Delta$ is to denote the symmetrical difference: $A \Delta B=$ $(A \backslash B) \cup(B \backslash A)$. Function Port gives the port corresponding to a given message. For a set $y$ of messages, function Ports is defined by: Ports $(y)=$ $\{k \mid \exists a \in y$ s. t. $k=\operatorname{Port}(a)\}$.

The local test sequences are basically projections of the complete test sequence over the port alphabets. In fact, Line 4 adds $! x_{i}$ to the sequence $\omega_{\operatorname{Port}\left(x_{i}\right)}$ 
and the loop of Line 5 adds the reception of messages belonging to $y_{i}$ to the appropriate sequences. Coordination messages are added to the projections to get the same controllability and observability when using the complete test sequence in centralized method.

Algorithm 1 uses two kinds of coordination messages: $C$ coordination messages for guaranteeing controllability, and $O$ coordination messages for guaranteeing observability.

As pointed out in [6], control problem may arise when the tester sending $x_{i+1}$ is neither the one sending $x_{i}$, nor one of those receiving a message belonging to $y_{i}$. In this case (Line 6), we add $+C$ and $-C$ to the appropriate local test sequences. $+C$ is added to $\omega_{h}$, where $h$ is the tester sending $x_{i+1} .-C$ is added to the sequence of a tester receiving a message belonging to $y_{i}$, if $y_{i} \neq \emptyset$ (Lines 8 and 9), if not $-C$ is added to the sequence of the tester sending $x_{i}$ (Line 7). The former case does not introduce any control problem because the reception of $C$ by tester $h$ means that $x_{i}$ has been received by IUT. However, in the latter case, one may still have a control problem if this exists in the centralized method because $x_{i+1}$ may be sent to the IUT before that $x_{i}$ has been received by it. Therefore, we have the same controllability as in centralized method.

Remark 1. Let us already notice that Lines 8 and 9 may also reduce the number of $O$ coordination messages. This is due to the fact that for sending $C$, Algorithm 1 considers if there exists a tester that receives a message belonging to $y_{i}$ and does not receive any message belonging to $y_{i+1}$.

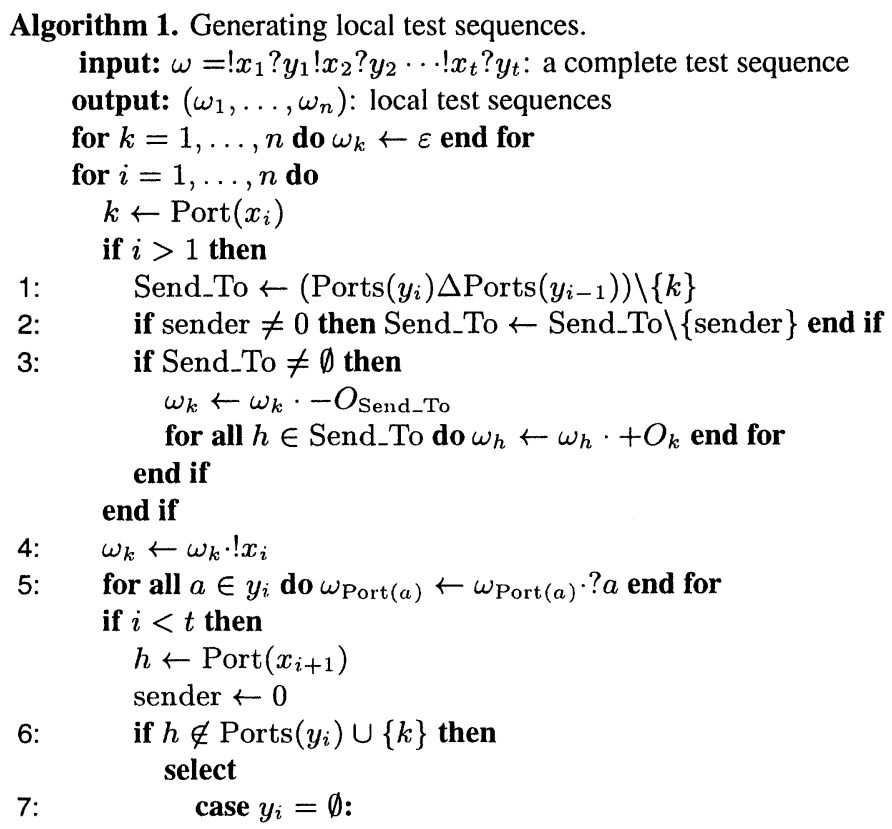


8:

$\omega_{k} \leftarrow \omega_{k} \cdot-C_{h}$

$\omega_{h} \leftarrow \omega_{h} \cdot+C_{k}$

sender $\leftarrow h$

case Ports $\left(y_{i}\right) \backslash \operatorname{Ports}\left(y_{i+1}\right) \neq \emptyset$ :

choose $l \in \operatorname{Ports}\left(y_{i}\right) \backslash \operatorname{Ports}\left(y_{i+1}\right)$

$\omega_{l} \leftarrow \omega_{l} \cdot-C_{h}$

$\omega_{h} \leftarrow \omega_{h} \cdot+C_{l}$

sender $\leftarrow l$

9:

otherwise:

choose $l \in \operatorname{Ports}\left(y_{i}\right)$

$\omega_{l} \leftarrow \omega_{l} \cdot-C_{h}$

$\omega_{h} \leftarrow \omega_{h} \cdot+C_{l}$

sender $\leftarrow l$

end select

end if

end if

end for

End Algorithm 1

To avoid observation problems, each tester receiving a message $a$ belonging to $y_{i-1}$ should be able to determine that $a$ has been sent by the IUT after the IUT has received $x_{i-1}$ and before the IUT receives $x_{i}$. This can be achieved by using an $O$ coordination message. $+O$ is added to the local test sequences of those testers receiving either a message belonging to $y_{i}$ or a message belonging to $y_{i-1} .-O$ is added to $\omega_{k}$ where $k$ is the tester sending $x_{i}$. It can be shown by induction [1] that this avoids observation problems. Finally, let us notice that Line 2 avoids sending $O$ to the tester having sent $C$ to tester $k$, reducing in this way the number of $O$ coordination messages as was said in Remark 1.

Example 4. Applying Algorithm 1 to test sequence (1), we get the following local test sequences:

$$
\left\{\begin{array}{l}
\omega_{1}=! a ? x ? x+O_{3}+C_{3} ! a ? x ? x ? w ! a ? x+O_{3} \\
\omega_{2}=? y ! b ? y-C_{3}+C_{3} ! b ? y-C_{3}+O_{3} ? y \\
\omega_{3}=+C_{2}-O_{1} ! c ? z-C_{1} ? z-C_{2}+C_{2} ! c ? z ? z-O_{\{1,2\}} ! c ? z
\end{array}\right.
$$

Using these sequences in the distributed method, one can detect all the faults of Figure 3.

\section{CORBA TESTING TOOLS}

The objectives of CORBA and ODP are quite similar. They aim to describe systems implemented in terms of configurations of distributed objects. To do that, they identify common infrastructure functions that support the operation of such systems and simplify their implementation. However if ODP is a generic model, CORBA is more implementation-oriented. Therefore, several implementations of CORBA are already commercially available. After introducing 
basic CORBA elements, this section is experiments with implementation of the distributed test method through a prototype within the CORBA infrastructure.

\subsection{CORBA}

To provide a standard for interoperability of distributed objects in heterogeneous environments, OMG has in fact developed two architectures: OMA (Object Management Architecture) and CORBA [7]. OMA defines its object terminology and various facilities required for object-oriented distributed processing. CORBA provides the mechanisms necessary to identify, locate, access and manipulate the OMA objects.

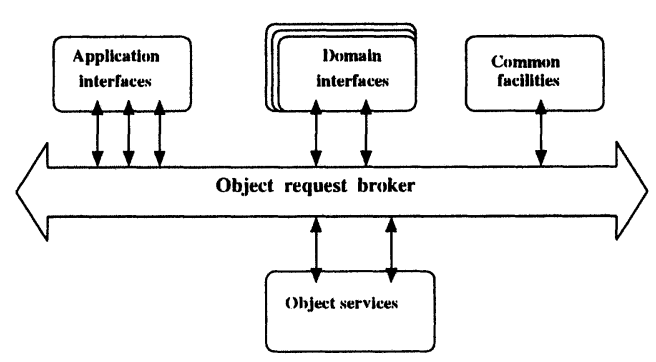

Figure 5. OMA reference architecture

OMA architecture. OMA is made up of an object model and a reference architecture. The object model defines how objects distributed across an heterogeneous environment can be described, while the reference architecture deals with interactions between those objects. Figure 5 shows the components of the OMA reference architecture, which are detailed below.

Object Request Broker (ORB): this is a kind of a message bus for communicating between objects of a distributed application regardless of their location and implementation.

Object services: These are domain-independent interfaces that provide fundamental assistance to application developers such as the naming and trading services. The naming service allows clients to find objects based on names while the trading service allows them to find objects based on their properties. They also include many other services such as event notification, transaction management and security.

Common facilities: These are a set of interfaces providing commonly required services to applications such as printing and compound documents.

Domain interfaces: A set of interfaces that are oriented towards specific application domains such as telecommunications manufacturing, and so on. 
Application interfaces: These are interfaces developed specifically for a given application.

CORBA specification. CORBA specification details the interfaces and characteristics of an ORB that conveys requests for invocation of objects operations from CORBA client to CORBA object implementation. Figure 6 shows the different an ORB interfaces. In CORBA, the terms client and server are used to specify the role played by a component in a distributed application. A client makes a request of some other component. A server provides an implementation of a component that a client uses. Servers may act as clients to other component. A pure client does not play the server role. Each component communicates with others on a peer-to-peer basis.

The key feature of an ORB is transparency. Usually, an ORB hides the following characteristics of objects: object location, object implementation, object execution state, and object communication mechanisms, by using object references. When a CORBA object is created, a corresponding object reference is also created. In other words, a creation request returns an object reference for the newly-created object to the client. To make a request, a client specifies the target object by using its object reference which is immutable and opaque.

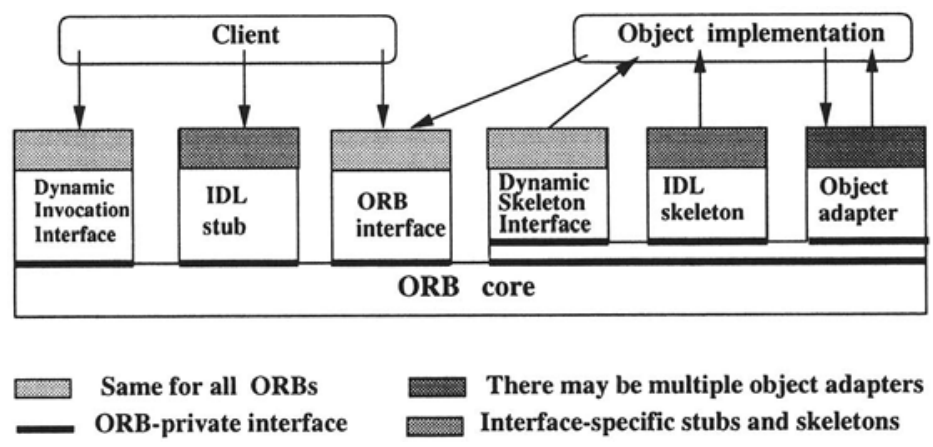

Figure 6. ORB interfaces

In CORBA, objects are defined only by their interfaces by using the OMG Interface Definition Language (IDL). An object interface specifies the operations and types that the object supports. Therefore, IDL is a purely declarative language. It is based on $\mathrm{C}++$ with additions for distribution. It does not provide features like control constructs, leaving one free to construct objects using different programming languages. OMG has defined several language mappings like C, C++, Smalltalk, Ada 95, Java and so on. These mappings are automatically implemented by IDL compilers, which generate code in the given programming language for the types specified in IDL and facilitate communication between clients and servers wherever they may be located. 
An ORB is drawn as a single entity in the OMA. Figure 6 shows that in fact it is implemented in several parts. In addition to generating programming language types, IDL compilers generate stubs and skeletons supporting what is called static invocation. A stub is a mechanism that creates and issues requests on behalf of client, while a skeleton delivers requests to the object implementation. CORBA also provides dynamic invocation through the dynamic invocation interface and dynamic skeleton interface, without generating stubs and skeletons at compile time. The ORB interface is to provide a variety of services from the ORB to clients and servers, and because the ORB core may be implemented in different ways an object adapter is required to provide standard interfaces to servers.

\subsection{PROTOTYPE STRUCTURE}

In developing a prototype for the distributed test method, three main testing aspects may be distinguished: preparation, execution and conclusion. Preparation consists of designing a test sequence for the IUT from its specification. Execution consists of connecting the testers to the IUT ports and providing them the related local test sequences. The testing can then be executed according to the behavior described in Section 3. Finally, the conclusion consists of collecting the testers verdicts to determine the global verdict. Figure 7 describes the prototype structure. It is made up of a set of testers, attached to the ports of the IUT and exchanging coordination messages through a multicast channel, called the Coordination Channel. A Management System is used for starting and ending the test as well as for determining the global test verdict. To do that, the Management System interacts with the testers through a specific channel called the Management Channel.

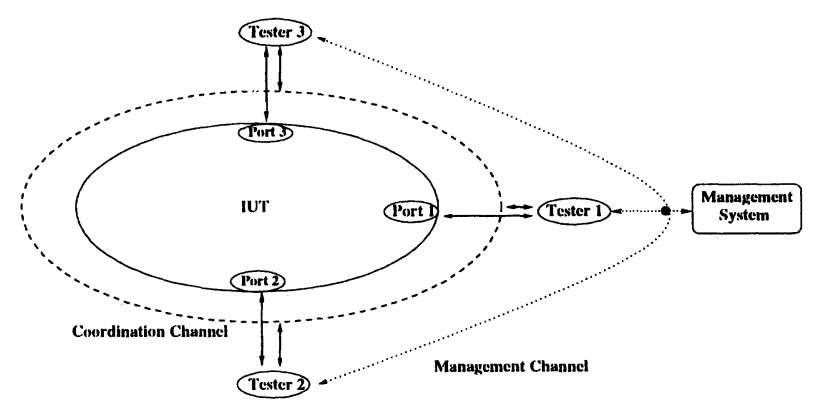

Figure 7. Prototype of distributed method 


\subsection{PROTOTYPE REALIZATION}

This section is dedicated to describe the main objects of the prototype, which are grouped into three categories: information objects, computing objects and communication objects.

Information objects. There are two types of information object: test sequences and verdicts.

A Test sequence object encapsulates the sequence of inputs to be applied to the IUT by a given tester as well as the expected results. It also includes the coordination messages to be sent to, or to be received from, other testers.

A Verdict object encapsulates information allowing the Management System to determine the global verdict. Each Tester object provides a local verdict to the Management System object.

Computing objects. There are two kinds of computing object: Tester object and Management System object.

A Tester object applies a test sequence to the IUT interface related to it and provides a local verdict. A Tester object behaves as described in Section 3.2.

A Management System object configurates the test application, dispatches the local test sequences, starts and ends the test by providing the user with a global verdict deduced from the local test verdicts.

Communication objects. Three kinds of communication are considered: communication between the Management system and the testers, communication between the testers, and communication between the testers and the IUT.

In communication between the Management system and the testers, three kinds of information are exchanged: commands to start and to end the test, local test sequences and local verdicts. It should be noticed that the local test sequences and the local verdicts are exchanged on a 1 to 1 basis: each tester receives its local test sequence and sends back its verdict. These exchanges can then be realized by method invocation through the ORB. Commands starting and ending the test are sent on 1 to $n$ basis: the management system must send them to each and every tester at the same time. This is realized through a broadcast channel (Management Channel) using the CORBA event service, which allows CORBA objects to produce events to be consumed by other objects. Commands are events produced by the Management System and consumed by the testers. Herein, the event service is used in push mode in which, when the producer puts an event into the channel, the consumers are automatically notified. Communication between testers is 1 to $n$ : a tester may send a coordination message to some or all other testers. This is realized through the Coordination Channel using the CORBA event service. Figure 8 


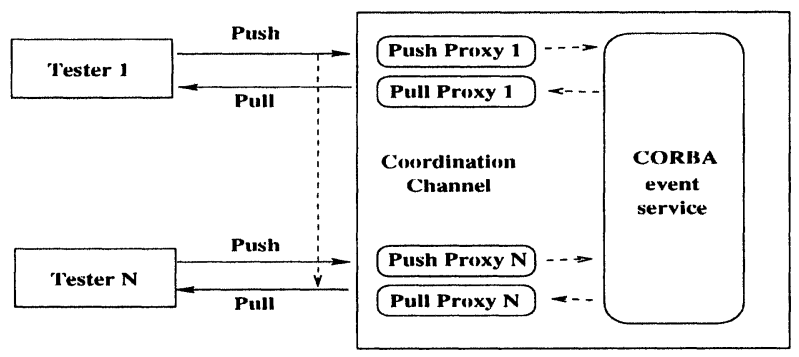

Figure 8. Coordination channel

gives the layout of the Coordination Channel. To send a coordination message to testers $h_{1}, \ldots, h_{r}$, tester $k$ invokes the Push method on object Push Proxy $k$. To receive it, tester $h_{i}$ invokes the Pull method on object Pull Proxy $h_{i}$. The coordination message is transfered from Push Proxy $k$ to Pull Proxy $h_{i}$ using the CORBA event service.

Communication between testers and the IUT consists mainly of me-thod invocation. To get a generic test application, adapting itself to the interfaces of the IUT, we introduce an Adapter object which provides a Tester object with methods to send and to receive actions. An Adapter object communicates with the associated IUT interface through the CORBA dynamic invocation mechanism.

\section{TESTING CARRYING OUT}

This section describes a testing example of a CORBA application using the test prototype. The application and its interfaces are briefly presented. Related local test sequences are constructed from a complete test sequence, and then test results are given and discussed.

\subsection{FORUM APPLICATION}

The application to be tested is a forum application, similar to a newsgroup where only subscribers can post and retrieve messages. The application has three interfaces:

- a User interface allowing subscribers to post and to retrieve messages;

- a MessageManager interface which is an extension of the User interface, allowing suppression of messages;

- a Manager interface allowing one to add and to remove subscribers. The IDL specification of these interfaces is given in Figure 9.

The $n$ p-FSM describing the behavior of the application is not given here, due to the space limitation in the paper. 

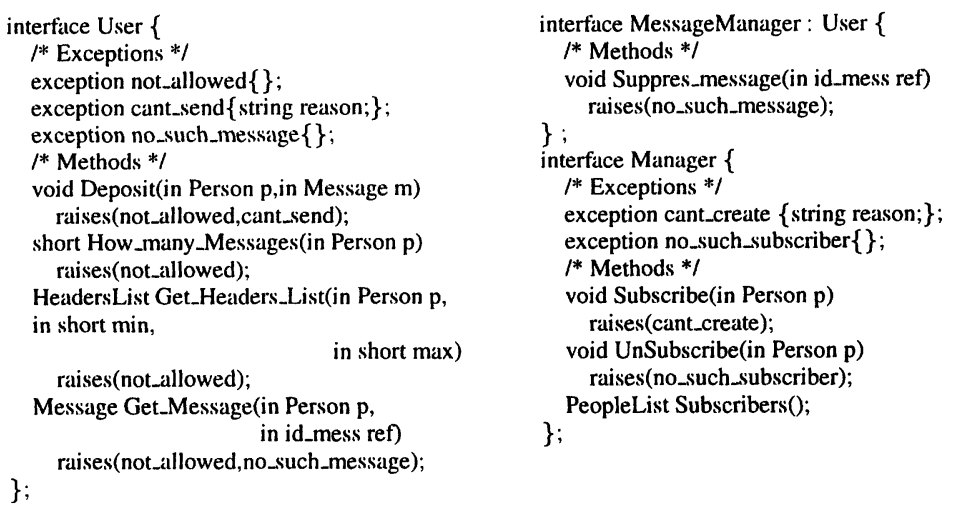

Figure 9. IDL interfaces of the forum application

\subsection{TEST EXECUTION}

To illustrate how local test sequences are constructed by the prototype, we use the test sequence of Figure 10. This sequence checks that a user who is not subscriber is not allowed to submit message. The notation used in Figure 10 is self explaining.

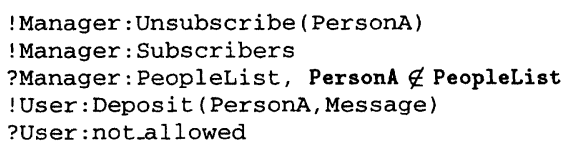

Figure 10. Test sequence of forum application

Local sequences corresponding to the sequence of Figure 10 are given in Figure 11. Notice that a coordination message is used to avoid PersonA sending a message before the Unsubscribe message has been received by the IUT.

\section{Manager}

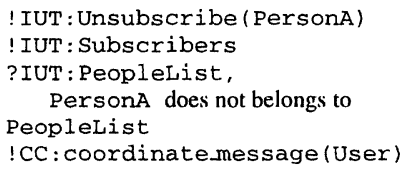

User

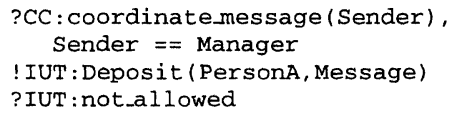

Figure 11. Local test sequences of Figure 10

Each tester uses two interfaces: IUT, related to the IUT interface associated with the tester, and $\mathrm{CC}$ related to the interface with the Coordination Channel. These sequences come from testing experiments with the prototype on students realizations of the forum application. 


\section{CONCLUSION AND FUTURE WORK}

With emergence of modern models and architectures such as ODP, CORBA and TINA, for developing new distributed applications, a related conformance testing methodology is required for getting working distributed systems. Our goal is to go from our experience with protocol testing to contributing to the design of such a framework. In this paper, we showed how OSI conformance test methods could be extended to take into account distributed systems providing more than two access points. After that, a distributed test method was designed and analyzed in terms of controllability and observability that play an important role in fault detection. A CORBA prototype of this method has been realized and experimented on students applications. Our work is now oriented to develop testing environments that are more adapted for tackling real-world applications.

\section{References}

[1] L. Cacciari and O. Rafiq, Controllability and observability in distributed testing, To appear in: Information and Software Technology.

[2] ISO/IEC, OSI conformance testing methodology and framework, 9646, Parts 1-7, 1991.

[3] ISO/IEC, ODP, Reference model, 10746, Parts 1-4, 1995.

[4] ISO/IEC, Information retrieval, transfer and management for OSI, Framework: formal methods in conformance testing, 1996.

[5] P. F. Linington, J. Derrick, and H. Bowman, The specification and testing of conformance in ODP systems, in: B. Baumgarten, H. J. Burkhardt and A. Giessler, eds, Testing of Communicating Systems, Chapman\&Hall, London, 1996, pp. 93-114.

[6] G. Luo, R. Dssouli, G. v. Bochmann, P. Venkataram, and A. Ghedamsi, Test generation with respect to distributed interfaces, in: Computer Standards and Interfaces 16, 1994, pp. 119-132.

[7] OMG, The common object request broker: architecture and specification, Version 2.2, 1998.

[8] A. Petrenko, G. v. Bochmann, and M. Yao, On fault coverage of tests for finite state specifications, in: Computer Networks and ISDN Systems 29, 1996, pp. 81-106.

[9] O. Rafiq, The Astride testing approach : principles, tools and carrying out, in: Computer Standards and Interfaces 11, 1990, 85-94.

[10] TINA-C, Overall concepts and principles of TINA, version 1.0, Document Label: TB_MDC.018_1.0_94, 1995. 